\title{
A COMPARISON OF MICROPATTERN GAS AVALANCHE DETECTORS
}

\author{
A. Sharma* \\ GSI-Darmstadt \\ GERMANY
}

\begin{abstract}
A large number of new micropattern detectors have emerged in the last few years, offering cheap and robust solutions for high luminosity tracking. A brief overview and comparison are presented.
\end{abstract}


Invited Talk at the 6th International Conference on Advanced Technology and Particle Physics, Como, Italy Oct. 5-9, 1998

\section{*Presently at CERN-EP, Geneva Switzerland} e-mail Archana.Sharma@cern.ch 


\section{Introduction}

What is required of the new generation of position sensitive gas micropattern avalanche detectors? Given the hostile environment for high luminosity tracking, these devices need to be very fast, fully efficient in detecting minimum ionizing particles (mips), and provide a good tracker energy and momentum resolution by employing their good spatial and two track resolutions. The very high radiation environment demands them to be non ageing, and sturdy in withstanding the interactions of highly ionizing particles. Activation of materials is a concern and eventually they need to be cheap due to the large detection surfaces needed. Simplicity of handling and manufacturing is a big advantage; some of these detectors have also found applications in other fields. Here I will trace their evolution in the last decade and present some comparisons of performance.

MicroStrip Gas Chambers (MSGCs) (see for eg. refs. [1]) have undergone rigorous tests in the last several years: good localization accuracies, double track and energy resolutions, full efficiencies for mips, rate capabilities and radiation tolerance are among their key performances leading their use in the CMS experiment at the LHC [2] and several other applied fields. Their drawback, apart from being fragile, are discharges resulting in irreversible damage to the anodes and cathodes in the presence of a flux of highly ionizing particles in addition to a high rate of mips. Several techniques have been proposed to salvage them, some of them will be discussed in section 6 and 7 .

\section{MICRODOT Gas Avalanche Chamber}

This detector was developed by the Liverpool group [3] and manufactured by employing the photolithography techniques of silicon foundry, it is essentially a true gaseous pixel device. It consists of cathode rings with an anode dot in the centre connected by an anode bus running at the bottom of a $5 \mu \mathrm{m}$ thick silicon dioxide layer, added to lower surface resistivity, sitting on the silicon wafer as shown in fig. 1(a). 


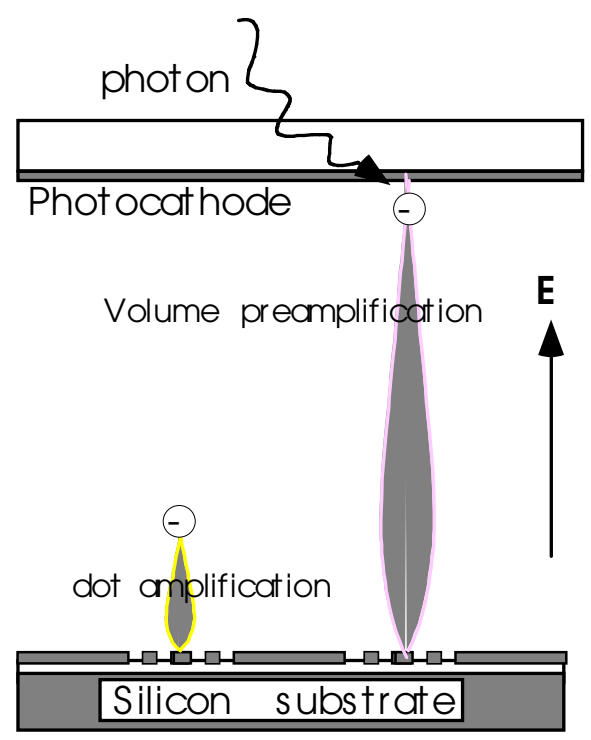

(a)

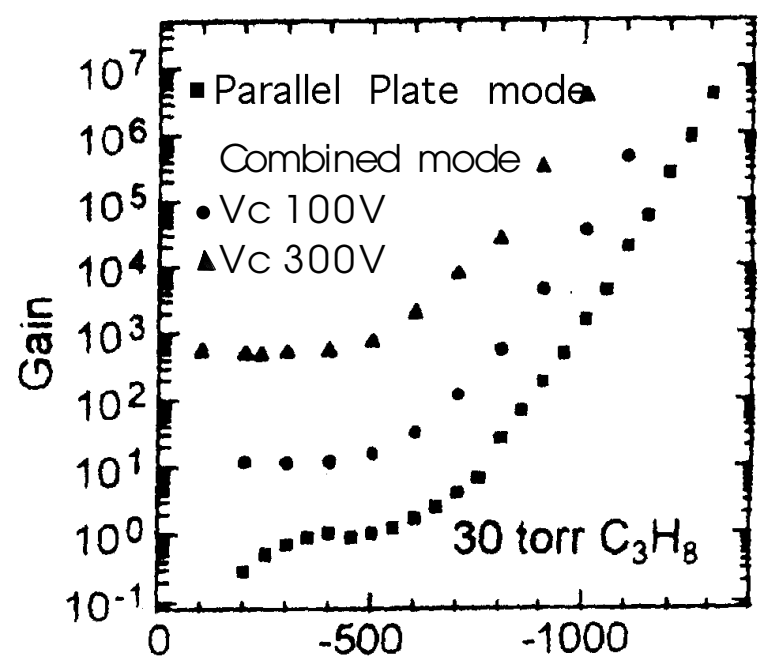

(b)

Fig. 1 (a) Scheme of a two stage amplification in a MICRODOT detector

(b) Gain in both parallel plate, and combined amlification

An additional cathode ring is placed between the cathode and the anode, such that the focusing effect of the anode bus on the electric field is minimized. This detector has yielded very high gains (few $10^{4}$ ) and was taken up by several groups; a low pressure operation in combination with a volume parallel plate preamplification was demonstrated in ref. [4] with extremely high gains $\left(10^{6}-10^{7}\right)$ as shown in fig. 1 (b) from [4]. With a photocathode placed on the underside of the drift plane this device is used for single photon detection.

\section{Gas Electron Multiplier (GEM)}

Introduced recently by Sauli [5], this device consists essentially of a Kapton foil ( 50 $\mu \mathrm{m})$ copper clad (several $\mu \mathrm{m})$ on both sides perforated by holes with typically $90-200 \mu \mathrm{m}$ pitch and 35-120 $\mu \mathrm{m}$ hole diameter made by a wet etching process used for printed circuit boards ${ }^{1}$. Upon application of a difference of potential between the two metallic surfaces the field at the centre of the hole exceeds $\sim 40 \mathrm{kV} / \mathrm{cm}$, sufficiently high for electron avalanche multiplication. 


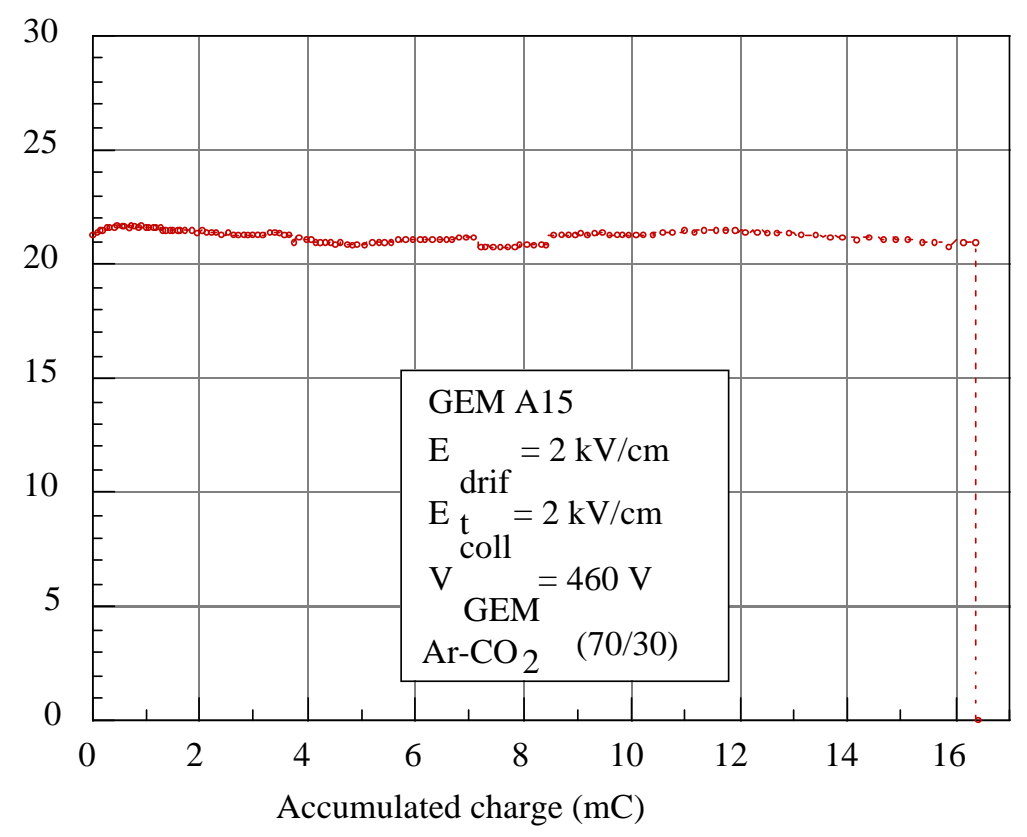

Fig. 2 Ageing measurements with a GEM [7].

Delimiting this foil by a drift cathode on the top and readout electrodes on the bottom, the device acts as a gas electron multiplier. A remarkable feature of this detector is that the readout is decoupled from the avalanche, hence there are no positive ions in the vicinity. Utilizing the experience gained from MSGCs in terms of light frames and materials, it has been demonstrated that this detector shows no ageing [6,7] as also demonstrated in fig. 2 from ref.[7]. Very high gains with GEMs have been reported see for example fig. 3(a); the gain of the GEM depends on the hole diameter as shown in fig. 3(b) from ref.[6]. This dependence being saturated around hole diameters ranging from 40-70 $\mu \mathrm{m}$, relaxes the precision on hole diameters in this range. GEMs have been demonstrated to be high rate capable and yield good single and two track resolutions $\sim 40 \mu \mathrm{m}$ and $200 \mu \mathrm{m}$ resectively. In view of lowering the material budget, very thin GEMs have also been manufactured having the metal layers of $\sim 200 \mathrm{~nm}$ of $\mathrm{Cr}-\mathrm{Ni}$, the so called adhesion layer ${ }^{2}$.

\footnotetext{
${ }^{2}$ GOULD Electronics Inc., Foil Division EASTLAKE, OH 44095-4001 USA
} 


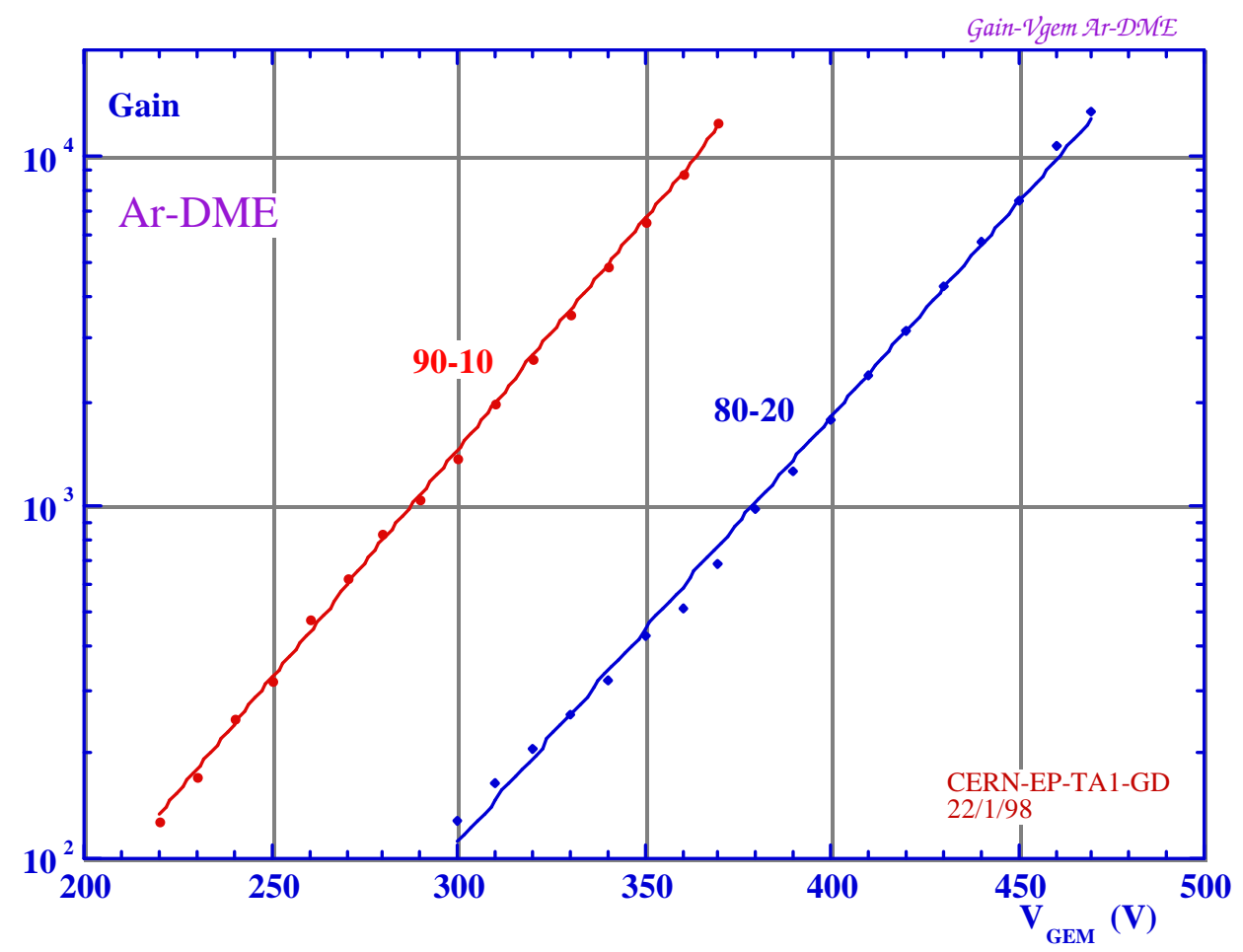

Fig. 3 (a) High gains with GEM in Ar-DME [6]

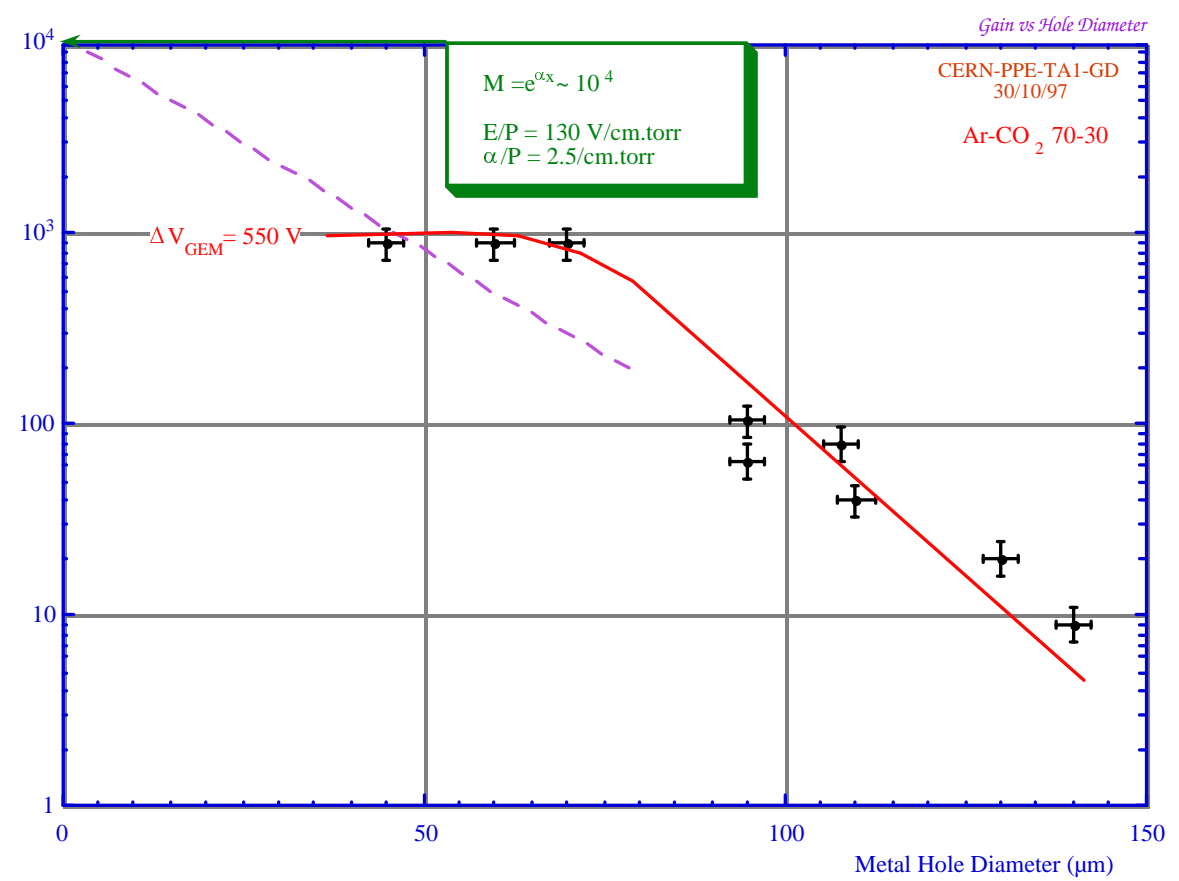

Fig. 3 (b) GEM amplification versus metal hole diameter [6] 


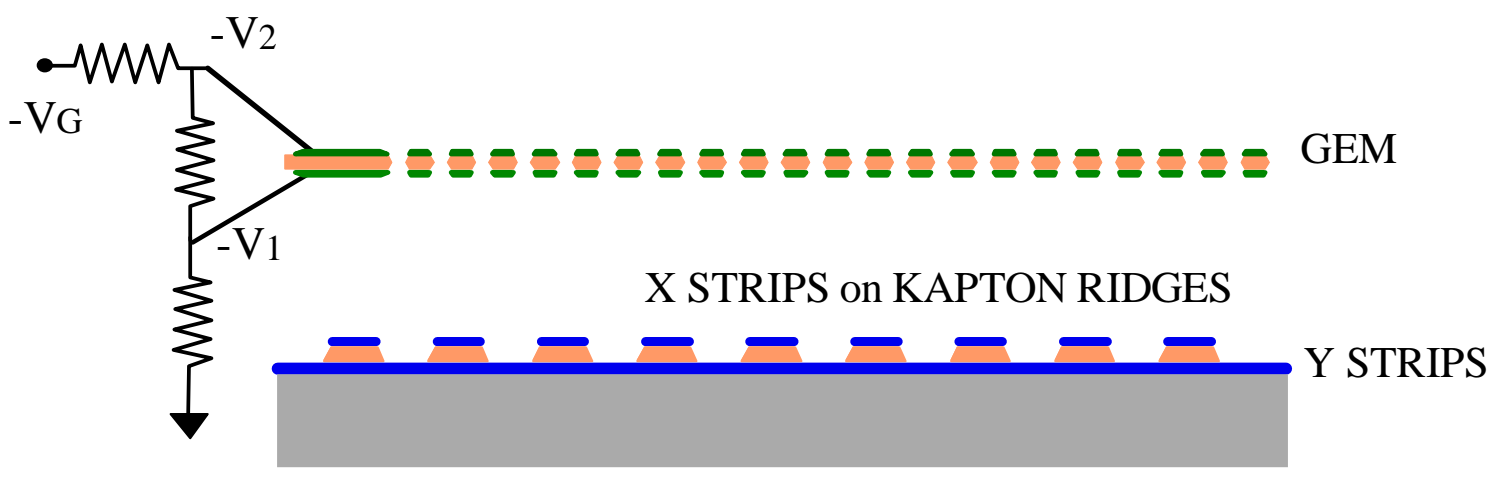

Fig. 4 Schematics of a two dimensional readout with A GEM detector

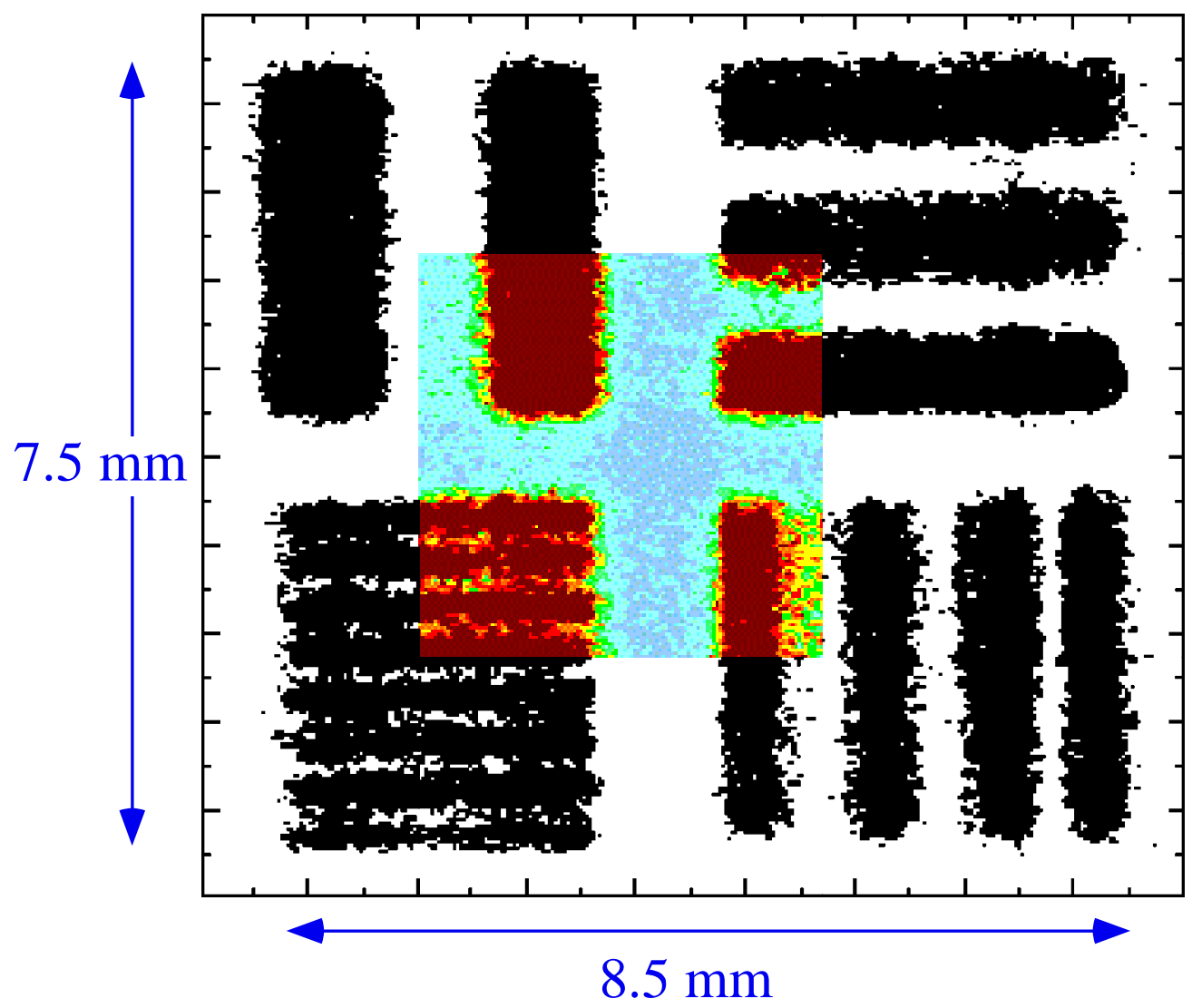

Fig. 5 Image obtained by transmission radiography with a GEM equipped with $2 \mathrm{~d}$ readout [10]. 
Two dimensional readout has been implemented [9] by manufacturing a 2D pickup circuit using the same technology as that for the GEMs, with X-strips on Kapton ridges and Y strips on the metal below, as shown in fig.4 from ref. [10]; several other readout patterns with Cartesian or small angle stereo are also discussed. An image obtained by transmission radiography of $8 \mathrm{keV} \mathrm{X-rays} \mathrm{is} \mathrm{shown} \mathrm{in} \mathrm{fig.5.} \mathrm{Using} \mathrm{wet} \mathrm{etching} \mathrm{as} \mathrm{the}$ technology of production, large surfaces of GEMs have been manufactured, the largest being 30 x $60 \mathrm{~cm}$, so called GEM banana for the forward CMS tracker.

\section{MICROMEGAS}

The MICROMEGAS detector has been introduced by Giomataris et al [11], and comprises a metallic mesh placed at a very small distance, 50-250 $\mu \mathrm{m}$, to the readout elements. With appropriate potentials applied, the electric field in this very thin gap, is $100 \mathrm{kV} / \mathrm{cm}$. Exploiting the semi-saturation of the Townsend coefficient at high fields, large gains $\left(10^{4}-10^{5}\right)$ are obtained in a range of gases. Fig. 6 shows the high rate capability of the detector withstanding rates of $10^{6} \mathrm{~Hz} / \mathrm{mm}^{2}$ at gains $10^{4}$. Typical spatial resolutions $50 \mu \mathrm{m}$ rms have been reported.

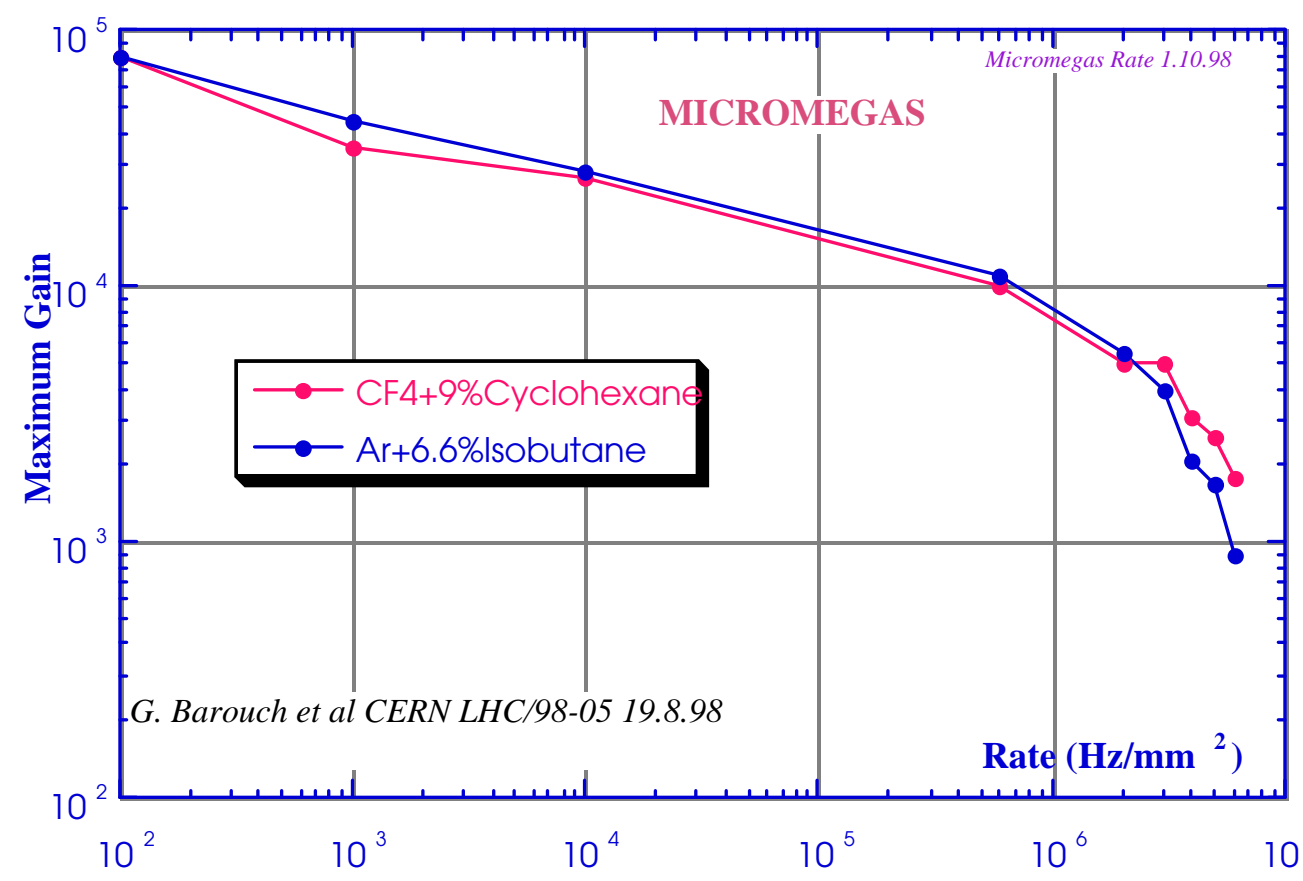

Fig. 6 Gain versus rate for the Micromegas detector [11]

\section{Compteur A Trou (CAT) and its derivatives}

The CAT detector proposed by Bartol et al [12] consists of a sandwich of a metallic cathode and anode separated by an insulator, with holes drilled through the cathode to the insulator. A drift plane and appropriate voltages yield a focusing of the filed lines in the hole, thus providing large gains for electrons deposited in the volume above. Gains $\sim 10^{4}$ 
have been demonstrated as shown in fig.7 from [12]. This detector was made by drilling, while the WELL detector, introduced by the PISA group [13], uses the technology developed by the printed circuit workshop at CERN for manufacturing the GEM detectors. The similar MICROGROOVE detector [14] has the edges of the insulator conical in shape to focus better the drift lines on the anodes, as shown in fig. 8. The $\mu \mathrm{CAT}$ detector studied by the Siegen group [15] has also shown very high gains and rate capabilities.

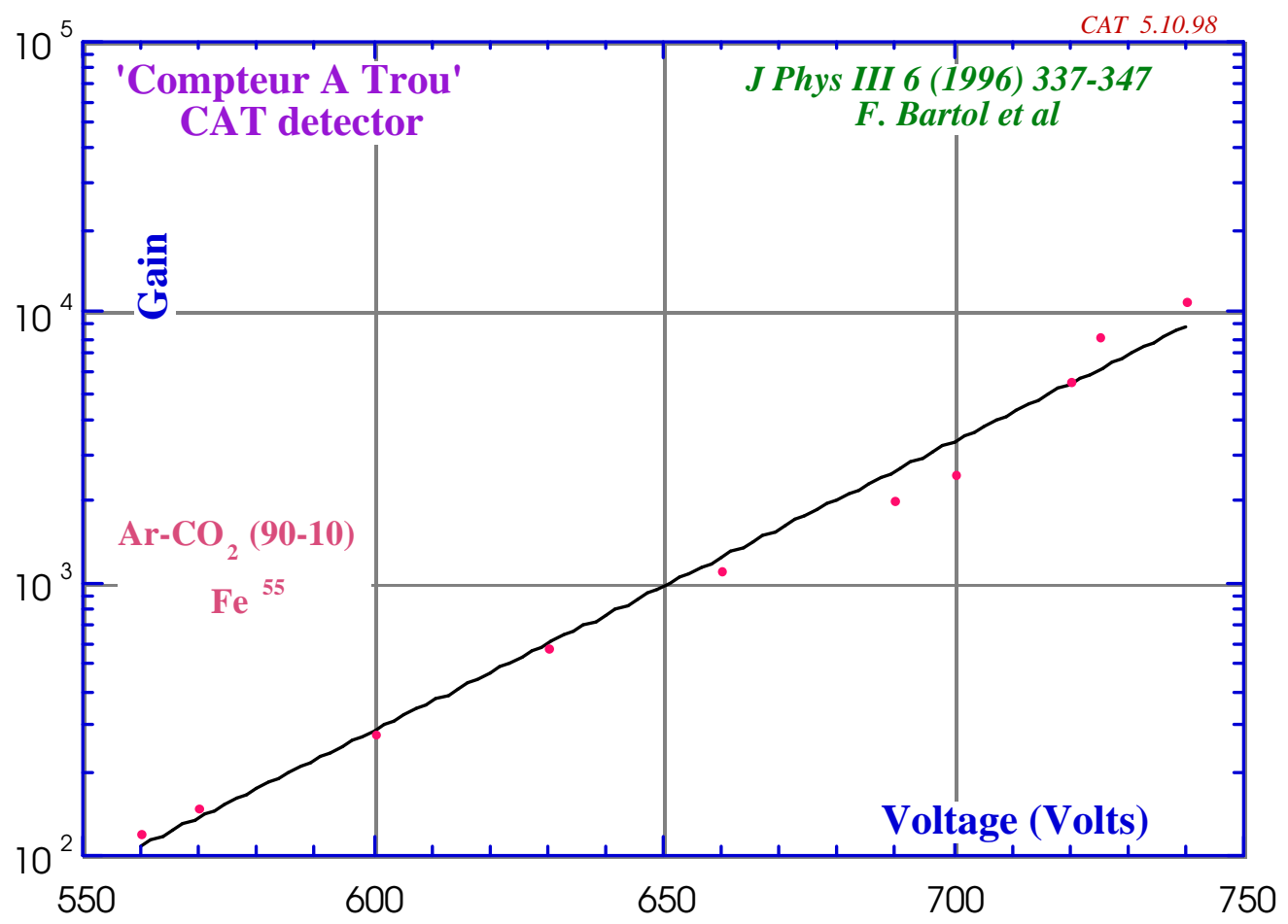

Fig. 7 Gain versus cathode voltage in a CAT detector [12] 


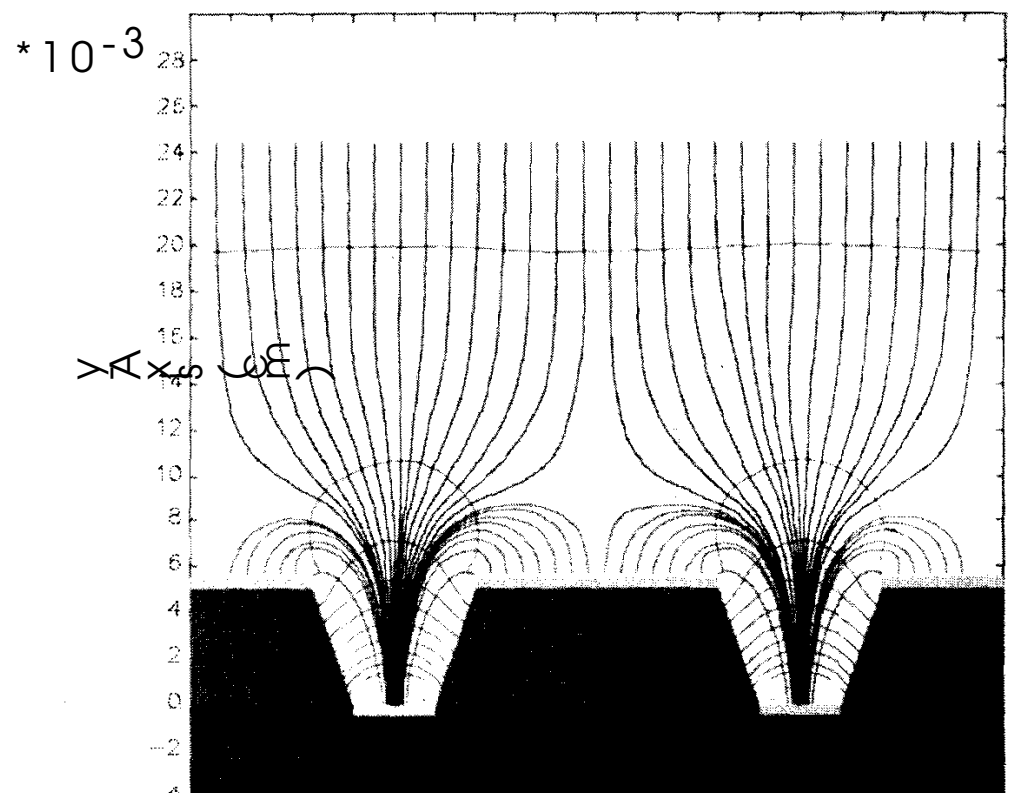

Fig. 8 Equipotential and drfit lines in a MICROGROOVE detector

\section{Discharge limits with heavily ionizing particles}

A detailed study of the performance of micropattern detectors in the presence of a high flux $\left(\sim 10^{4} \mathrm{~Hz} / \mathrm{mm}^{2}\right)$ of minimum ionizing particles accompanied by heavily ionizing particles like alphas of few $\mathrm{MeV}$, was undertaken by several groups to simulate the fierce environment expected at hadron colliders. MSGCs suffered irreversible damages when exposed to such radiation [16,17]: their fragile surface quality, higher gains due to higher electric field at cathode edges, and electron emissions and spontaneous transitions from avalanche to streamers and microdischarges being responsible.

Several techniques were proposed to overcome this problem. The Virtual Cathode Chamber (VCC) [18] was introduced eliminating all the cathodes from the MSGC surface and defining the field by a backplane potential. Though the principle was successfully demonstrated, this detector warrants an electron conducting substrate which is not easily available ${ }^{3}$. Another possibility was to decouple the current limitation and signal speed by using two different metals as anodes and cathodes [19], nevertheless requiring two masks for the production. Introduced by T. Tanimori et al [20], and taken up by the PISA group [21] passivation of the cathode strip edges was also proposed as a way to eliminate discharges (so called advanced passivation). A similar albeit cheaper option was the small gap chamber (SGC) [22], where all the surface between the anodes and cathodes is covered by polyimide, also reducing their spacing.

\section{Two stage Amplification}

\footnotetext{
${ }^{3}$ The VCC tested was made by the BINP Novosibirsk, Russian Federation
} 
The concept of dividing the total detector gain into two sub critical stages was introduced by Sauli [5]. Combining the GEM and MSGC a two stage amplification detector was assembled and successfully tested for high rate capability, operation in a magnetic field of $1 \mathrm{~T}$ [6], and with large signal to noise in test beams [23], see for example fig. 9 from [24]. Ageing tests with no gain drop have been reported in refs. [23,25]. This solution despite its obvious success, adopted by the HERA-B group [19], nevertheless comprises the fragile and expensive MSGCs. An elegant solution was to cascade the GEM in two stages in the so called DOUBLE GEM detector [23]. This detector has been shown to withstand high rates; results of recent beam tests with high resolutions, full efficiency of mips detection and signal to noise up to 1000 have been reported in [23].

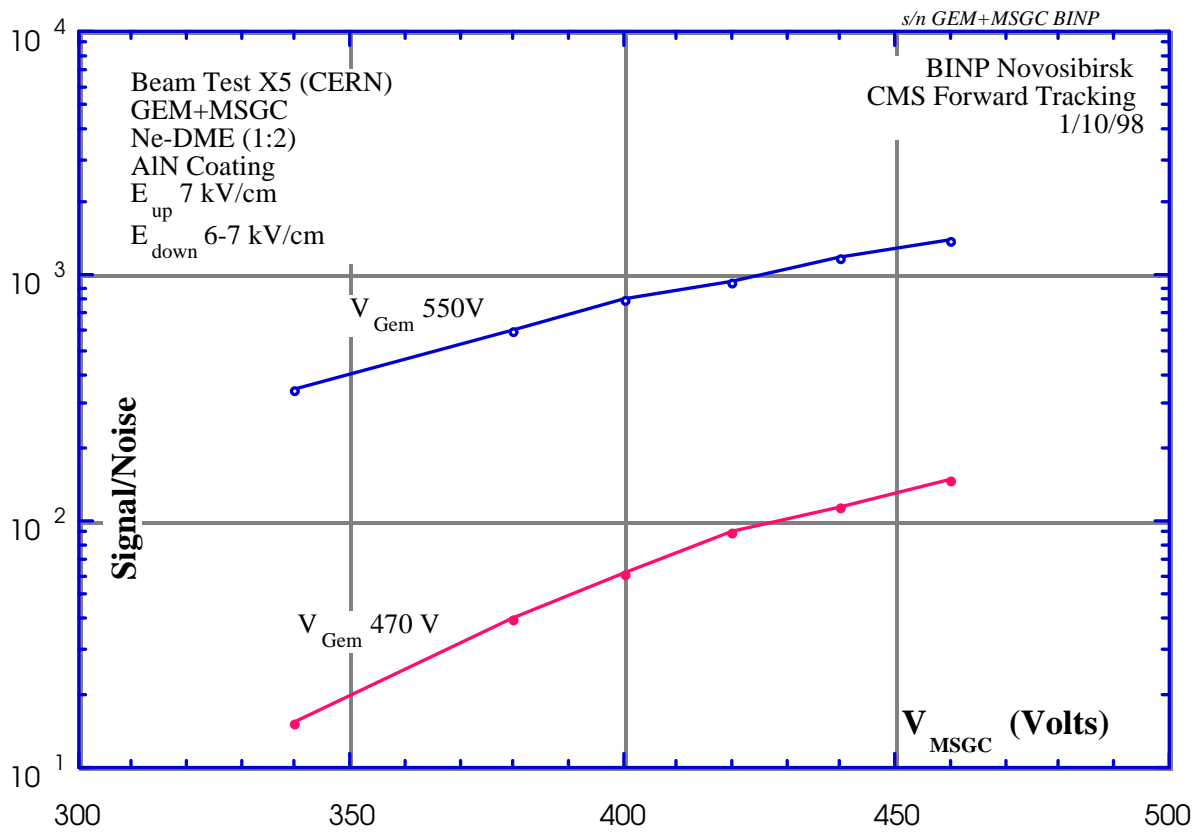

Fig. 9 Signal/noise versus voltage on the MSGC at two voltages on the GEM [23] 


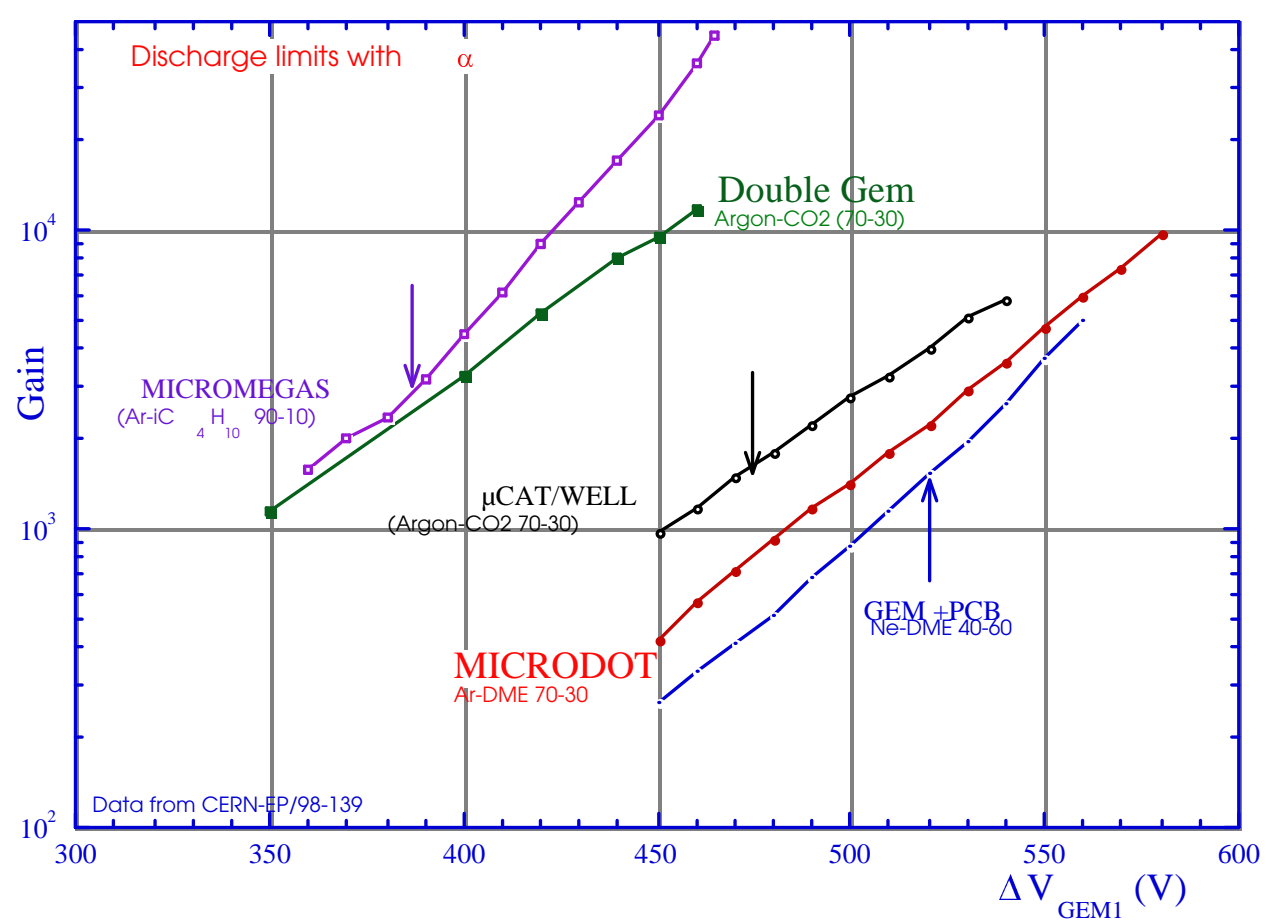

Fig. 10 Discharge limits for some micropattern detectors with high mip flux in the presence of highly ionizing particles

- Vdrift

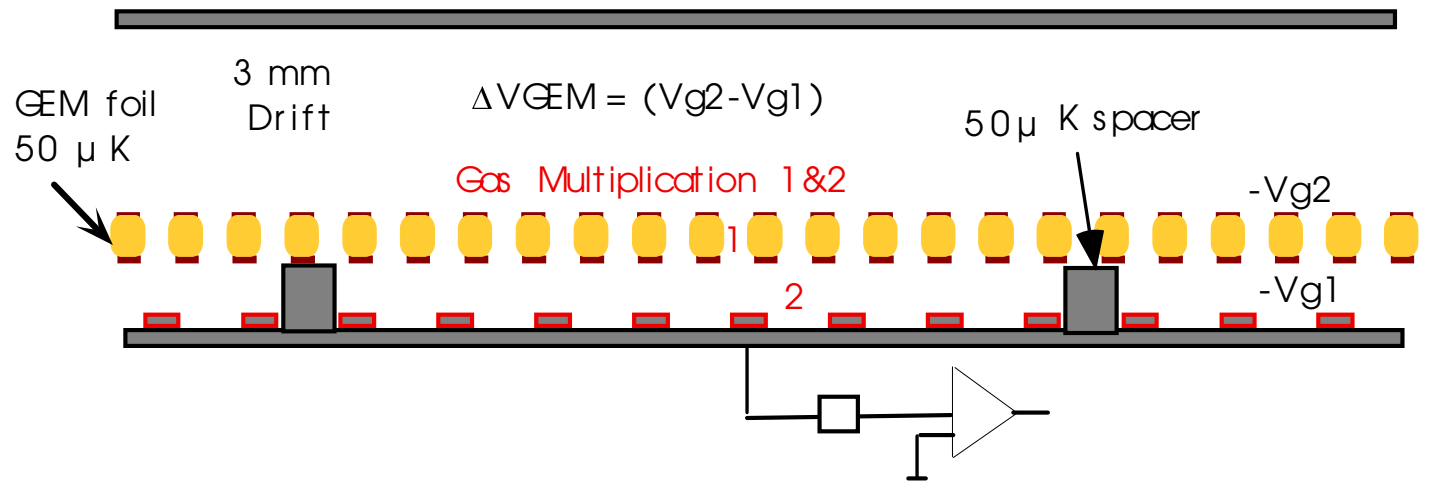

Fig. 11 Scheme of the MICROMEGEM (GEM operated in Micromegas mode)

Fig. 10 shows the performance of a number of these micropattern detectors, in the presence of a high flux of X-rays and simultaneously alphas from an internal Rn source. Details of the measurement may be found in [17]. The arrows represent the voltages and gains at which breakdown occurs. It may be noted that except for the MICRODOT detector, all single stage amplification detectors reach discharge limits around a gas gain of few thousands. The authors confirm voltage dependence of the Raether limit (avalanche size $10^{7}$ ) at which breakdown occurs; due to lower voltages in two stage amplifications, the detectors do not reach this limit despite the same total gain. This limit seems to be independent of the gas mixture used. Another two stage amplification device, 
the so called MICROMEGEM combining the high gains of GEM and MICROMEGAS detectors, has been introduced by Van Doninck [26], with a scheme shown in fig. 10. Spacers of $50 \mu \mathrm{m}$ have been incorporated in the readout electrode made by the printed circuit technology, and a GEM is placed on top, thus having a very high electric field between GEM and the readout strips. This detector has also attained large gains as shown in fig. 11 [27]. Similar structures have been tested by the PISA group, placing GEMs over MICROGROOVEs yielding very high gains and two dimensional readout capabilities [28], shown to operate in a high rate and heavily ionizing environment with no dangers of discharges.

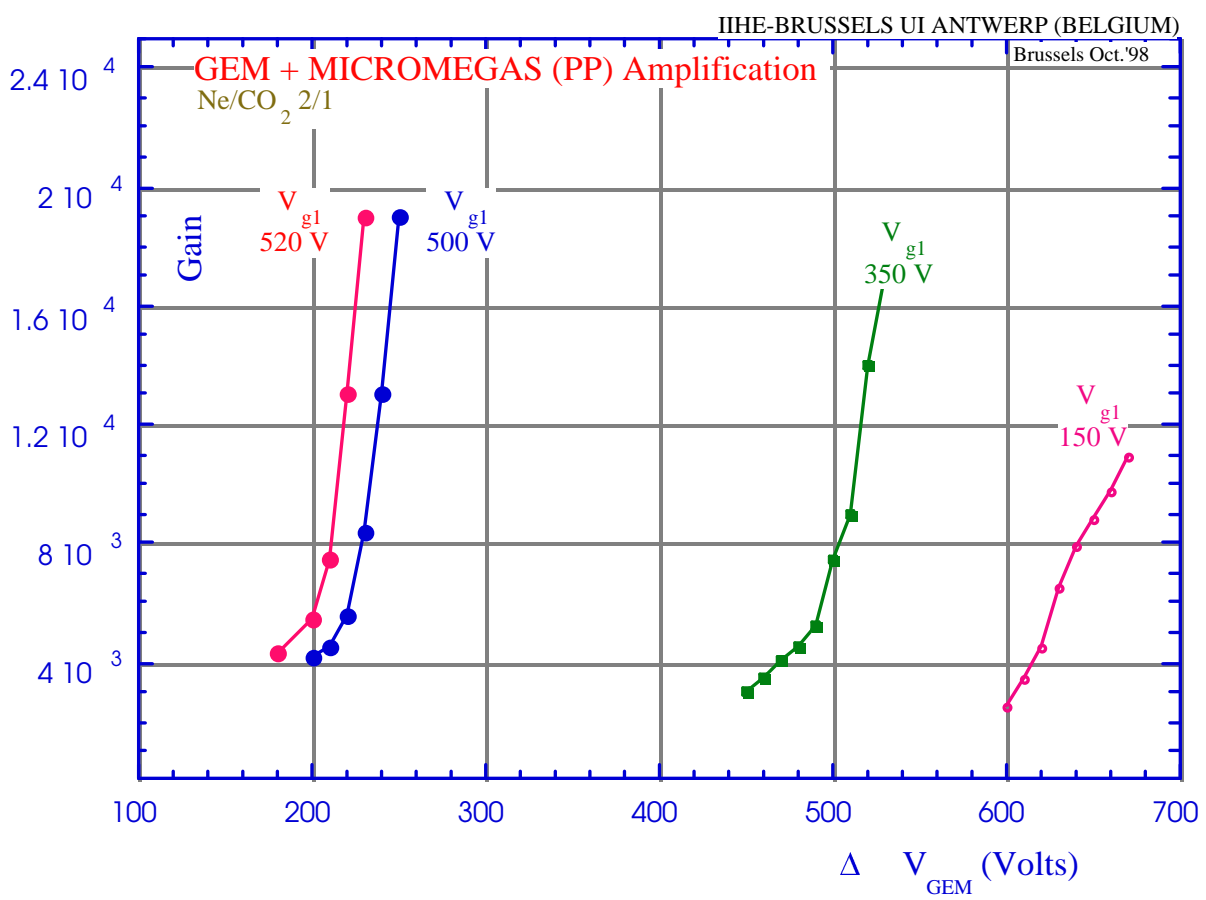

Fig. 12 Gain characteristics [[26,27] of MICROMEGEM; see fig. 10 for scheme

\section{Conclusions and Discussion}

In the last few years there has been considerable progress in the field of micropattern gas detectors resulting from the advancement in microelectronics photolithography and printed circuit board technologies. The MSGC and its derivatives have been thoroughly studied and progressively developed. Full efficiencies for mips, good space and two track resolution, high rates, no ageing and no loss in performances in magnetic field have been reported by various groups. Yet they have gain limitations in a highly ionizing environment, due to their fragility and necessary high fields across the substrate. Additionally they are quite expensive for large area high luminosity trackers. The single stage CAT, $\mu$ CAT, GEM, WELL, GROOVE and MICROMEGAS kind of detectors have also outstanding performance in terms of gains, resolutions, high rate capabilities, full efficiencies, operation in magnetic fields and 2-d imaging. They are also cheap and robust, and have been manufactured in large sizes. Their limitation still being the 
discharge limits at lower gain, not yielding sufficient margins of operation. The two stage amplification concept solves the discharge limit problems, with the MSGC+GEM being the repaired and expensive approach, while the DOUBLE GEM or GEM $+\mathrm{X}$, where $\mathrm{X}$ is any micropattern foil seems to be the sturdy solution for tracking at forthcoming hadron colliders. With some experience in the field of ageing on these detectors, the materials may be carefully chosen, nevertheless long term ageing tests, operation in high magnetic fields, and activation of the metal foils remains to be investigated.

\section{References}

[1] A. Oed, Nucl. Instrum. Methods A263 (1988) 351, See for example: Proc. International Workshop on Micro-Strip Gas Chambers, Legnaro, Italy, (1994)

Proc. Int. Workshop on Micro-Strip Gas Chambers, Lyon (1995)

F. Angelini et al., Nucl. Instr. and Meth. A 382 (1996) 461-469,

Proc. of the Wire Chamber Conference, Vienna, 1998

[2] The CMS Experiment for the LHC Collider, CERN LHCC94-38

[3] S.F. Biagi and T.J. Jones, Nucl. Instrum. Methods A361 (1995) 72

[4] R. Chechik, Contribution to Wire Chamber Conference, Vienna 1998

[5] F. Sauli, Nucl. Instr. and Meth. A 386 (1997)531

[6] J. Benlloch et al, Wire Chamber Conference Vienna, 1998. CERN-EP/98-50

[7] J. A. Kadyk, Private Communication, September 1998

[8] C. Buttner et al, et al CERN-PPE/97-73

[9] A. Bressan et al, in preparation October 1998

[10] A. Bressan, contribution to this conference

[11] Y. Giomataris, DSM DAPNIA/SED 95-04, CERN/LHC/96-04 May 1996, G. Barouch et al CERN LHC/98-05 19.8.98

[12] F. Bartol et al, J. Phys. III 6(1996)337-347

[13] R. Bellazzini et al [], INFN PI/AE 98/03

[14] R. Bellazzini et al [], INFN PI/AE 98/02

[15] A. Sarvestani et al, contribution to this conference

[16] R. Bouclier et al CMS-TN/96-018 (1996)

[17] B. Boimska et al, CMS-TN/97-020 (1997); Nuclear Physics B 61B (1998) 498; B. Schmidt (see [19]), V. Peskov et al LIP Report 98/03, Proceedings Vienna

Wire Chamber Conference, 1998

[18] M. Capeans et al CERN-PPE/97-61, Nucl. Instr. and Meth. A 400(1997)17-23

[19] B. Schmidt, Proceedings Vienna Wire Chamber Conference, 1998

[20] T. Tanimori, Nucl. Instr. and Meth. A381 (1992) 280

[21] R. Bellazzini, CMS Document 1996-146 (1996)

[22] D. Contardo, Proceedings Vienna Wire Chamber Conference, 1998

[23] A. Bressan et al, CERN-EP/98-139, Submitted NIM, Sept. 1998

[24] T. Fukimoto, contribution to this conference

[25] Measurements by the BINP Forward CMS group, Oct. 1998, Private Comm. A. Buzulutzkov

[26] W. VanDonink private communication, Sept. 1998 
[27] Measurements done by V. Zhukov IIHE-Brussels, UI-Antwerp, Belgium, Private Comm., 1998

[28] R. Bellazini et al, contribution to this conference. 